\title{
Re-Justification for Reducing the Length of Hospital Stay Based on Community-Based Appraisal of Functional Recovery of Cerebrovascular Disease Patient
}

\author{
Kazuaki Kuwabara $^{\mathrm{a}, \mathrm{g}}$, Shinya Matsuda ${ }^{\mathrm{b}}$, Kiyohide Fushimic, Koichi B Ishikawa ${ }^{\mathrm{d}}$, \\ Hiromasa Horiguchie, Kenji Fujimori ${ }^{f}$
}

\begin{abstract}
Background: Because longer length of hospital stays (LOS) have been recognized to pose a significant economic burden on society, attempts to reduce LOS in acute care hospitals have been implemented to improve the efficiency of care. However, few studies have quantitatively measured the inherent effect of LOS on patient functional recovery in acute care settings. To determine whether a reduction in LOS is justified, we tested whether a longer LOS is associated with the deterioration of functional recovery in patients with nontraumatic cerebrovascular disease (CVD).
\end{abstract}

Methods: Using Japanese administrative data collected between 2004 and 2008 across 138 acute care hospitals, 19,175 CVD patients with alert consciousness at admission and at discharge were included to remove the sequence of longer LOS caused by consciousness deterioration during hospitalization. The Barthel index (BI) at admission as well as the difference between the BI at admission and discharge were examined. Patient and hospital character-

Manuscript accepted for publication April 29, 2011

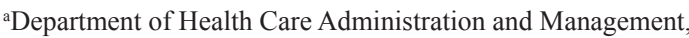
Graduate School of Medical Sciences, Kyushu University, 3-1-1 Maidashi, Higashi-ku, Fukuoka 812-8582, Japan

${ }^{b}$ Department of Preventive Medicine and Community Health, University of Occupational and Environmental Health, 1-1 Iseigaoka,

Yahatanishi-ku, Kitakyushu, Fukuoka, Japan

'Department of Health Policy, Section of Healthcare Informatics,

Graduate School of Medicine, Tokyo Medical and Dental University,

1-5-45 Yushima, Bunkyo-ku, Tokyo, Japan

dStatistics and Cancer Control Division, National Cancer Center,

5-1-1 Tsukiji, Chuo-ku, Tokyo, Japan

eDepartment of Health Management and Policy, Graduate School

of Medicine, The University of Tokyo, 7-3-1 Hongo, Bunkyo-ku, Tokyo, Japan

${ }^{\mathrm{f}}$ Division of Medical Management, Hokkaido University, 5 Nishi, 14

Kita, Kita-ku, Sapporo, Hokkaido, Japan

${ }^{g}$ Corresponding author: Kazuaki Kuwabara,

E-mail: kazu228@basil.ocn.ne.jp

doi:10.4021/jnr18e istics, fiscal year, LOS, and total charges (TC; $\$ 1=100$ yen) were analyzed among three BI improvement categories (deterioration, no change, and improvement). The effects of longer LOS and age on change in BI were determined using mixed linear regression models where study hospitals were handled as a random intercept. To respond to the critique of handling the ordinal variable of $\mathrm{BI}$ as the continuous one, logistic regression was employed to assess the association of longer LOS with BI deterioration.

Results: Three point five percent (3.5\%), 59.7\%, and $36.9 \%$ of patients experienced deterioration, no change, and improvement in $\mathrm{BI}$, respectively, and $55.5 \%, 88.9 \%$, and $15.6 \%$ of patients in these respective groups were nearly completely independent at admission. The mean age of deterioration, no change, and improvement in BI was 73.7, 66.5, and 69.5 years, respectively. Longer LOS was significantly associated with less BI improvement, which was more remarkable in patients $\geq 75$ years of age. Rehabilitation was not observed to determine the degree of BI improvement, but delayed rehabilitation was observed to predict BI deterioration.

Conclusions: Because a longer LOS was not observed to ameliorate functional recovery, stakeholders should determine the appropriate LOS in acute CVD care and monitor unnecessary hospitalization, especially in the elderly.

Keywords: Cerebrovascular disease; Functional recovery; Length of hospital stay; Rehabilitation

\section{Introduction}

Modern society is experiencing an aging population. Political stakeholders have triggered debates regarding the efficient allocation and financing of healthcare delivery in developed countries [1]. In 2004, approximately $15 \%$ of the Japanese population was at least 70 years of age and accounted for $40.6 \%$ (US $\$ 130$ billion) of national healthcare expenditures. Furthermore, patients aged 70 years or more with cerebrovascular disease (CVD) accounted for US \$12 billion in healthcare expenditures [2]. It is well known that this age group is likely to suffer from non-communicable and resource-consuming diseases including cancer, CVD, 
and cardiovascular diseases, and that disease management is a major focus of research for disease prevention and rehabilitation after acute onset of these diseases [3]. Health service research has long been performed to profile the economic burden on patients, their families and on society, and has focused on efficient resource use because the discharge rate per 100,000 population is not decreasing [4].

To reduce this burden, attempts have been made to reduce the length of hospital stay (LOS) to enhance the quality or efficiency of care by implementing rehabilitation. Many factors have been debated to explain the variation in LOS by adjusting for patient characteristics such as age and stroke severity; treatment strategies or system organizations such as interdisciplinary care delivery; and hospital characteristics such as hospital volume [1, 5-10]. Before focusing on healthcare resource utilization, patient outcomes and quality of daily life should be also considered as priorities. Therein, age disparity, volume-quality relationships, and variations in case mixes have often been enrolled in that consideration.

In recognition of these conditions, the rationale for reducing LOS should be confirmed. To enhance the efficiency of stroke care and eliminate healthcare costs, referral policies for rehabilitation facilities and home-based settings have been implemented, which force institutions to reduce hospitalization and to provide an effective strategy aimed at recovering the functional activity of daily life $[7,9,11]$. However, care-related health outcomes other than mortality and complications have not been sufficiently investigated to justify reducing $\operatorname{LOS}[1,5,12]$. Indeed, few quantitative analyses have estimated the original effect of longer LOS on functional status in acute care settings [8]. Hospitalization, although necessary for effective care after an acute event, removes patients from their daily lifestyle. Patients need to lie on beds, which may lead to functional impairment, especially in the elderly with limited residual physiological capacity. Community-based studies investigating the relationship between longer LOS spent in acute care hospitals and functional recovery during hospitalization should help to validate policies monitoring unnecessary LOS or advocating the strengthening of hospital functions, including patient referral to long-term care facilities and home care to provide more comfortable environments familiar to the patient.

Using a Japanese administrative database, we tested whether longer hospitalization was associated with deterioration in the Barthel index (BI), a proxy for the patient's recovery of activities of daily life (ADL), and identified the factors associated with the observed BI improvements.

\section{Methods}

\section{Data}

This was a secondary data analysis conducted as part of a government-funded research project on the development of a Japanese case-mix classification system. Anonymous claims and clinical data were gathered from 1,428 hospitals (82 academic hospitals and 1,346 community hospitals) by the Ministry of Health, Labour and Welfare (MHLW) until 2008. The data, including patients' daily care processes, were collected by the MHLW and merged into a standardized electronic format for this project over 4 - 6 month periods every year since July 1, 2002. Of a total of 8,010,361 patients across 1006 hospitals participating in our research, we analyzed patients with nontraumatic CVD and alert consciousness at admission and at discharge, who were consecutively treated in 151 hospitals between fiscal years (FY) 2004 and 2008. To distill out the inherent impact of longer LOS on change in functional status, we attempted to eliminate either the cases requiring a longer LOS sequent to consciousness deterioration during admission or those with consciousness deterioration observed during the longer hospitalization. Our project was approved by the ethical committee of the University of Occupational and Environmental Health, Fukuoka, Japan.

\section{Study variables}

Study variables included age, sex, principal diagnosis, use of an ambulance, outcome at discharge, destination (home or another facility), disability status, weighted comorbidity, type of procedure defined in a case-mix definition table, procedure-related complications, use of ventilation as a proxy of the influence of intensive care stay on ADL and rehabilitation, quantity (days) or initiation of rehabilitation during hospitalization, hospital teaching status (academic or community), patient volume of study hospitals, FY, LOS, and total charge (US $\$ 1=100$ yen). Age was stratified into 4 categories: 15 - 65 years, $65-74$ years, $75-84$ years, and $\geq$ 85 years. Emergency admission was defined as transport to hospital by an ambulance. The principal diagnosis of CVD was recorded using the appropriate International Classification of Diseases 10th Revision codes and categorized as transient ischemia (TIA; G45), lacunar status (G46), intracranial hemorrhage (I61), subarachnoid hemorrhage (SAH; I60), subdural hematoma (I62), infarction (I63), and reversible ischemic neurological deficits (I65-6). A maximum of 4 comorbidities and 4 complications were recorded in the database. Weighted comorbidity status was calculated using the Charlson comorbidity index (CCI), which includes dementia and CVDs [13]. These 2 conditions were also examined individually. The procedures for CVDs of interest were identified and analyzed, and included percutaneous endovascular interventions (e.g., coil implantation, angioplasty, or thrombolysis), carotid endarterectomy, clipping, decompression craniotomy, and evacuation of intracranial hematoma. In terms of complications, deep vein thrombosis (DVT) and/or pulmonary embolism and procedure-related complications were examined. The latter included wound complications, 
Table 1. Patient Characteristics and Outcomes by Barthel Index Improvement Category

\begin{tabular}{|c|c|c|c|c|c|}
\hline & & Deterioration & No change & Improvement & $\mathbf{P}$ \\
\hline \multicolumn{2}{|l|}{ Overall } & 668 & 11440 & 7067 & \\
\hline \multicolumn{6}{|l|}{ Age } \\
\hline & Mean age [SD] & $73.7[11.2]$ & $66.5[13.2]$ & $69.5[12.4]$ & $<0.001$ \\
\hline & $65-74$ years & $186(27.8)$ & 3652 (31.9) & $2138(30.3)$ & $<0.001$ \\
\hline & 75 - 84 years & $246(36.8)$ & 2618 (22.9) & 2065 (29.2) & \\
\hline & 85 years and over & $108(16.2)$ & $655(5.7)$ & $644(9.1)$ & \\
\hline \multicolumn{6}{|l|}{ Gender } \\
\hline & Male & $370(55.4)$ & $7561(66.1)$ & $4256(60.2)$ & $<0.001$ \\
\hline Transfer & not at home & $329(49.3)$ & $957(8.4)$ & $1582(22.4)$ & $<0.001$ \\
\hline Ambulance & use & $184(27.5)$ & 1918 (16.8) & $2658(37.6)$ & $<0.001$ \\
\hline \multicolumn{6}{|c|}{ Principal disease } \\
\hline & Transient ischemia & $26(3.9)$ & 1252 (10.9) & $563(8)$ & $<0.001$ \\
\hline & Lacunar infarction & $129(19.3)$ & $1613(14.1)$ & $1237(17.5)$ & \\
\hline & $\mathrm{SAH}$ & $13(1.9)$ & $286(2.5)$ & $269(3.8)$ & \\
\hline & Hemorrhage & $47(7)$ & $619(5.4)$ & $706(10)$ & \\
\hline & Nontraumatic subdural hematoma & $36(5.4)$ & $580(5.1)$ & $418(5.9)$ & \\
\hline & Infarction & $370(55.4)$ & $4801(42)$ & $3542(50.1)$ & \\
\hline & RIND & $47(7)$ & $2289(20)$ & $332(4.7)$ & \\
\hline \multicolumn{6}{|c|}{$\mathrm{BI}$ at admission } \\
\hline & Dependent & $189(28.3)$ & $982(8.6)$ & $4174(59.1)$ & $<0.001$ \\
\hline & Assisted independence & $108(16.2)$ & $285(2.5)$ & $1789(25.3)$ & \\
\hline & Independence with minimal assistance & $371(55.5)$ & $10173(88.9)$ & $1104(15.6)$ & \\
\hline \multicolumn{5}{|c|}{ Charlson comorbidity index } & $<0.001$ \\
\hline & 1 & $188(28.1)$ & $3043(26.6)$ & $1898(26.9)$ & $<0.001$ \\
\hline & 2 & $106(15.9)$ & 1249 (10.9) & $1006(14.2)$ & \\
\hline & 3 & $40(6)$ & $363(3.2)$ & $349(4.9)$ & \\
\hline & 4 or more & $19(2.8)$ & $134(1.2)$ & $125(1.8)$ & \\
\hline \multicolumn{2}{|c|}{ Preexisting cognitive impairment } & $2(0.3)$ & $0(0)$ & $5(0.1)$ & $<0.001$ \\
\hline \multicolumn{2}{|c|}{ Preexisting cerebrovascular disease } & $111(16.6)$ & $1835(16)$ & $1041(14.7)$ & 0.044 \\
\hline
\end{tabular}

RIND: reversible ischemic neurological deficit; SAH: subarachnoid hemorrhage; BI: Barthel index; SD: standard deviation. 
Table 1. Patient Characteristics and Outcomes by Barthel Index Improvement Category (Continued)

\begin{tabular}{lllll}
\hline & Deterioration & No change & Improvement & P \\
\hline Atrial fibrillation or flutter & $52(7.8)$ & $522(4.6)$ & $477(6.7)$ & $<0.001$ \\
Procedure-related complication & $8(1.2)$ & $112(1)$ & $60(0.8)$ & 0.524 \\
Cognitive impairment after admission & $3(0.4)$ & $31(0.3)$ & $22(0.3)$ & 0.660 \\
Deep vein thrombosis and pulmonary embolism & $1(0.1)$ & $20(0.2)$ & $15(0.2)$ & 0.827
\end{tabular}

Procedure

Surgical procedure

\begin{tabular}{|c|c|c|}
\hline Present & $135(20.2)$ & 1905 (16.7) \\
\hline
\end{tabular}

Ventilation

\begin{tabular}{|c|c|c|c|}
\hline Administered & $10(1.5)$ & $126(1.1)$ & $60(0.8)$ \\
\hline Mean days administered [SD] & $2.8[3.6]$ & $3.9[5.2]$ & $3.8[7.3]$ \\
\hline
\end{tabular}

Rehabilitation

$\begin{array}{lcccc}\text { Administered } & 398(59.6) & 3392(29.7) & 3769(53.3) & <0.001 \\ \text { Mean days administered [SD] } & 16.0[17.6] & 10.1[12.9] & 12.9[13.9] & <0.001\end{array}$

Hospital

Patient volume

\begin{tabular}{|c|c|c|c|}
\hline LVH & $212(31.7)$ & $4233(37.0)$ & $2087(29.5)$ \\
\hline IVH & $205(30.7)$ & 3998 (34.9) & 2279 (32.2) \\
\hline HVH & $251(37.6)$ & $3209(28.1)$ & $2701(38.2)$ \\
\hline
\end{tabular}

Teaching status

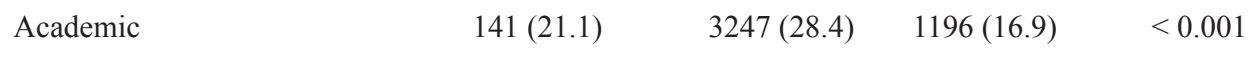

Fiscal year

$\begin{array}{llll}2004 & 90(3.8) & 1505(63.4) & 779(32.8)\end{array} \quad<0.001$

Resource use

Mean LOS, days [SD]

$\begin{array}{llll}27.6[25.1] & 13.9[14.2] & 19.4[16.7] & <0.001 \\ 10362[8779] & 5948[6199] & 7706[6867] & <0.001\end{array}$

Mean TC, \$ [SD]

LVH: low-volume hospital; IVH: intermediate-volume hospital; HVH: high-volume hospital; LOS: length of hospital stay; TC: total charge; SD: standard deviation. 
hematoma, and laceration or disruption of the treated organs by instrumentation or manipulation, for example, (T81 T87) [14]. Cognitive impairment after admission, which was defined in the CCI, was also examined [13].

Disability status was assessed by the BI score at admission and at discharge. BI at admission was grouped into the following categories: $<59=$ dependent; $60-84=$ assisted independence; and $\geq 85$ = nearly complete independence [15]. Changes in BI score were determined as BI at discharge minus $\mathrm{BI}$ at admission if the improvement was more than zero and categorized into 3 groups: improvement, no change, and deterioration [3]. Hospital volume and academic status were examined as hospital characteristics. The former was categorized into high-, intermediate-, or low-volume hospitals (HVH, IVH, or LVH), with each group containing an equal number of patients. Academic hospitals included university hospitals and the National Cardiovascular Center involved in educating medical students and postgraduate trainees and delivering clinical research. The dependent variables were changes in BI. Deceased patients were excluded from this analysis.

\section{Statistical analysis}

Proportions and descriptive statistics within each BI category were compared using Fisher's exact test and analysis of variance. Mixed linear regression models were used to identify the impact of longer LOS on change in BI score. BI has a ceiling effect, and BI category at admission was included in the models [10]. To control for hospital location and differences in hospital practices, the study hospital was included as a random intercept in the 3 models. An interaction between LOS and age categories and between LOS and study CVDs was also included in Model 2 and 3, respectively. The number of days of rehabilitation and day of rehabilitation initiation, which were weakly to moderately correlated with LOS (Spearman's correlation coefficient: $0.522(\mathrm{P}<0.001)$ and $0.266(\mathrm{P}<0.001)$, respectively), were included as covariates in all 3 models because we believe that a more explicit impact of LOS, a proxy of bed rest, on BI improvement should be extracted to adjust for the effect of rehabilitation. Spearman's correlation coefficient between rehabilitation day and day of rehabilitation initiation was -0.374 ( $\mathrm{P}<0.001$ ). To overcome the critique of handling the ordinal variable of $\mathrm{BI}$ as the continuous one, we employed logistic regression analysis to assess the relationship between longer LOS and BI deterioration. All statistical analyses were performed using SPSS version 16.0. All P-values were two-tailed, and the level of significance was set at $<0.05$.

\section{Results}

Among 45,502 CVD patients across 151 acute care hospitals
(41 academic and 110 community hospitals), 19175 CVD patients with alert consciousness both at admission and discharge were identified across 138 hospitals (32 academic and 106 community hospitals).

Overall, 668 patients $(3.5 \%)$ experienced a deterioration in $\mathrm{BI}, 11,440$ (59.7\%) experienced no change, and 7,067 $(36.8 \%)$ experienced an improvement in BI. The mean age was statistically different among the BI change categories (deterioration, 73.7 years; no change, 66.5 years; improvement, 69.5 years; $\mathrm{P}<0.001)$. The patient characteristics and care processes received differed significantly among the BI categories with the exception of procedure-related complications, cognitive impairment after admission, and DVT. Surgical procedures and rehabilitation were significantly more frequently used in patients with BI deterioration. BI deterioration and improvement were more frequently observed in HVHs than in LVHs and IVHs. There was a statistical difference in the proportion of patients experiencing improvements in BI among the study FYs. Mean LOS and mean TC were 27.6 days and US $\$ 10,362$, respectively, for patients showing deteriorations in $\mathrm{BI} ; 13.9$ days and $\$ 5,948$, respectively, for patients with no change in BI; and 19.4 days and $\$ 7,706$, respectively, for patients showing improvements in BI (Table 1).

Age $\geq 65$ years and advancing age categories were significantly associated with less BI improvement in any study model. Study comorbidities or complications, CCI 1, 3 and 4 , patient volume, and teaching status were not significantly associated with BI improvement. Significant BI improvement was observed in FYs 2007 and 2008. The interaction between LOS and age categories and between LOS and study principal diagnosis was observed with the exception of TIA and hematoma (Table 2).

Longer LOS and delayed initiation of rehabilitation were significantly associated with BI deterioration, whereas more rehabilitation was not (Table 3).

\section{Discussion}

This study revealed a quantitative association between LOS and the magnitude of BI improvement, based on a community-based appraisal of patients with nontraumatic CVD in Japanese acute care hospitals. We found that longer hospitalization was likely to worsen functional recovery in all study models and age categories. The initiation of rehabilitation, quantity of rehabilitation day, and hospital characteristics were not observed to determine functional improvement.

The Japanese healthcare system has been praised for its effects on health status [16]. Meanwhile, the mean LOS for acute care hospital admissions decreased from 24.8 days in 2000 to 19.2 days in 2006, but was still 2 to 4 times longer than that in several Organization for Economic Co-operation and Development (OECD) countries in 2006 [4]. The 
Table 2. Factors Associated With Improvement of Barthel Index

\begin{tabular}{|c|c|c|c|c|c|c|}
\hline Independent variables & \multicolumn{2}{|c|}{$\begin{array}{c}\text { Model 1 } \\
\text { Estimation 95\% CI }\end{array}$} & \multicolumn{2}{|c|}{$\begin{array}{c}\text { Model 2 } \\
\text { Estimation 95\% CI }\end{array}$} & \multicolumn{2}{|c|}{$\begin{array}{c}\text { Model 3 } \\
\text { Estimation 95\% CI }\end{array}$} \\
\hline Intercept & 51.6 & {$[46.7-56.4]$} & 49.8 & {$[44.8-54.7]$} & 50.5 & {$[44.5-56.5]$} \\
\hline \multicolumn{7}{|l|}{ Age (for $15-64$ years) } \\
\hline $65-74$ years & -2.0 & {$[-3.2--0.8]$} & -0.4 & {$[-2.3-1.4]$} & -2.0 & {$[-3.2--0.8]$} \\
\hline $75-84$ years & -4.8 & {$[-6.0--3.5]$} & -2.1 & {$[-4.0--0.1]$} & -4.8 & {$[-6.0--3.5]$} \\
\hline 85 years and over & -13.3 & {$[-15.2--11.4]$} & -10.6 & {$[-13.4--7.8]$} & -13.3 & {$[-15.1--11.4]$} \\
\hline Male & 0.4 & {$[-0.6-1.4]$} & 0.3 & {$[-0.7-1.3]$} & 0.3 & {$[-0.7-1.3]$} \\
\hline Transfer not at home & -16.2 & {$[-17.5--15.0]$} & -16.1 & {$[-17.4--14.9]$} & -16.2 & {$[-17.5--15.0]$} \\
\hline Ambulance & 4.0 & {$[2.9-5.1]$} & 4.0 & {$[2.9-5.1]$} & 4.0 & {$[2.8-5.1]$} \\
\hline \multicolumn{7}{|l|}{ Principal Diagnosis (for TIA) } \\
\hline Lacunar & -1.0 & {$[-4.7-2.7]$} & -1.0 & {$[-4.8-2.7]$} & -0.6 & {$[-5.9-4.7]$} \\
\hline SAH & 16.6 & {$[11.6-21.6]$} & 16.4 & {$[11.3-21.4]$} & 20.8 & {$[13.3-28.4]$} \\
\hline Hemorrhage & 3.0 & {$[-0.9-6.9]$} & 2.9 & {$[-1-6.8]$} & 0.3 & {$[-5.3-6.0]$} \\
\hline Subdural hematoma & -2.2 & {$[-7.2-2.8]$} & -2.1 & {$[-7.1-3.0]$} & 3.8 & {$[-3.4-11.0]$} \\
\hline Infarction & -0.7 & {$[-4.3-2.9]$} & -0.7 & {$[-4.4-2.9]$} & 0.4 & {$[-4.7-5.5]$} \\
\hline RIND & 0.2 & {$[-4.0-4.4]$} & 0.2 & {$[-4.0-4.4]$} & 3.2 & {$[-3.1-9.5]$} \\
\hline \multicolumn{7}{|l|}{ BI at admission (for dependent) } \\
\hline Assisted independence & -24.7 & {$[-26.2--23.2]$} & -24.7 & {$[-26.2--23.2]$} & -24.7 & {$[-26.2--23.2]$} \\
\hline Independence with minimal assistance & -46.3 & {$[-47.6--45.1]$} & -46.2 & {$[-47.4--45]$} & -46.3 & {$[-47.5--45.1]$} \\
\hline \multicolumn{7}{|l|}{ Charlson comorbidity index (for zero) } \\
\hline 1 & -1.0 & {$[-2.2-0.3]$} & -0.9 & {$[-2.1-0.3]$} & -1.0 & {$[-2.2-0.3]$} \\
\hline 2 & -1.4 & {$[-2.8-0.0]$} & -1.4 & {$[-2.9-0.0]$} & -1.4 & {$[-2.9-0.0]$} \\
\hline 3 & -1.3 & {$[-3.5-0.8]$} & -1.3 & {$[-3.4-0.9]$} & -1.4 & {$[-3.6-0.8]$} \\
\hline 4 or more & -3.2 & {$[-6.6-0.1]$} & -3.1 & {$[-6.5-0.3]$} & -3.3 & {$[-6.6-0.1]$} \\
\hline Cerebral vascular accident & -0.2 & {$[-1.7-1.4]$} & -0.1 & {$[-1.6-1.4]$} & -0.1 & {$[-1.7-1.4]$} \\
\hline Dementia & -10.3 & {$[-26.8-6.3]$} & -10.3 & {$[-26.9-6.2]$} & -10.2 & {$[-26.7-6.4]$} \\
\hline
\end{tabular}

Cl: confidence interval; RIND: reversible ischemic neurological deficit; SAH: subarachnoid hemorrhage; $\mathrm{BI}$ : Barthel index.

OECD acknowledged that the reason for the longer LOS was partly because of the broader differentiation of "acute care" compared with the definition used in other OECD countries [17]. Ironically, these circumstances specific to Japan have enabled studies, such as ours, to be performed. Discussions regarding the efficiency of care and differentiation of hospital function might be appropriate because longer hospitalization and rehabilitation in acute care hospitals do not always accompany better quality of care for CVD. Thus, the present study might be validated once patients in other OECD coun- 
Table 2. Factors Associated With Improvement of Barthel Index (Continued)

\begin{tabular}{|c|c|c|c|c|c|c|}
\hline \multirow{2}{*}{$\begin{array}{l}\text { Independent variables } \\
\text { Atrial fibrillation or flutter }\end{array}$} & \multicolumn{2}{|c|}{$\begin{array}{c}\text { Model } 1 \\
\text { Estimation 95\% CI }\end{array}$} & \multicolumn{2}{|c|}{$\begin{array}{c}\text { Model 2 } \\
\text { Estimation 95\% CI }\end{array}$} & \multicolumn{2}{|c|}{$\begin{array}{c}\text { Model } 3 \\
\text { Estimation 95\% CI }\end{array}$} \\
\hline & -1.2 & {$[-3.1-0.6]$} & -1.2 & {$[-3.0-0.7]$} & -1.2 & {$[-3.1-0.7]$} \\
\hline Procedure-related complication & -2.7 & {$[-8.6-3.3]$} & -2.3 & {$[-8.3-3.6]$} & -3.0 & {$[-9.0-3.0]$} \\
\hline Cognitive impairment after admission & -1.3 & {$[-9.2-6.5]$} & -0.9 & {$[-8.7-7.0]$} & -1.0 & {$[-8.9-6.8]$} \\
\hline Deep vein thrombosis & -0.3 & {$[-9.4-8.8]$} & -0.2 & {$[-9.4-8.9]$} & -0.7 & {$[-9.8-8.4]$} \\
\hline Procedure (reference; Medical) & -1.8 & {$[-3.8-0.2]$} & -2.0 & {$[-4-0.0]$} & -2.2 & {$[-4.2--0.1]$} \\
\hline Ventilation & -4.0 & {$[-8.3-0.3]$} & -4.5 & {$[-8.8--0.2]$} & -3.6 & {$[-8.0-0.7]$} \\
\hline \multicolumn{7}{|l|}{ Patient volume (reference; LVH) } \\
\hline IVH & -1.4 & {$[-3.7-0.9]$} & -1.4 & {$[-3.8-0.9]$} & -1.4 & {$[-3.7-0.9]$} \\
\hline $\mathrm{HVH}$ & 0.1 & {$[-2.6-2.9]$} & 0.1 & {$[-2.8-2.9]$} & 0.2 & {$[-2.7-3.0]$} \\
\hline \multicolumn{7}{|l|}{ Hospital (reference; Community) } \\
\hline Academic & -1.2 & {$[-3.6-1.2]$} & -1.2 & {$[-3.7-1.2]$} & -1.2 & {$[-3.6-1.2]$} \\
\hline \multicolumn{7}{|l|}{ Fiscal year (for 2004) } \\
\hline 2005 & 1.5 & {$[-0.7-3.7]$} & 1.6 & {$[-0.7-3.8]$} & 1.5 & {$[-0.7-3.7]$} \\
\hline 2006 & 1.6 & {$[-0.7-3.8]$} & 1.7 & {$[-0.5-4.0]$} & 1.7 & {$[-0.5-4.0]$} \\
\hline 2007 & 2.4 & {$[0.1-4.7]$} & 2.5 & {$[0.2-4.9]$} & 2.6 & {$[0.2-4.9]$} \\
\hline 2008 & 3.0 & {$[0.6-5.3]$} & 3.1 & {$[0.8-5.5]$} & 3.2 & {$[0.8-5.5]$} \\
\hline \multirow[t]{7}{*}{ LOS, one more day } & \multicolumn{2}{|c|}{$-0.174[-0.230--0.118]$} & a -0.104 & {$[-0.172--0.037]$} & e -0.114 & {$[-0.357-0.129]$} \\
\hline & & & b -0.174 & {$[-0.243--0.106]$} & f -0.152 & {$[-0.230--0.074]$} \\
\hline & & & ${ }^{c}-0.224$ & {$[-0.293--0.156]$} & $\mathrm{g}-0.250$ & {$[-0.371--0.130]$} \\
\hline & & & $\mathrm{d}-0.221$ & {$[-0.306--0.136]$} & h -0.032 & {$[-0.124-0.061]$} \\
\hline & & & & & ${ }^{\mathrm{i}}-0.417$ & {$[-0.610--0.225]$} \\
\hline & & & & & $\mathrm{j}-0.181$ & {$[-0.240--0.121]$} \\
\hline & & & & & ${ }^{k}-0.252$ & {$[-0.386--0.118]$} \\
\hline Initiation of rehabilitaion, one day delayed & 0.075 & {$[0.002-0.147]$} & 0.083 & {$[-0.001-0.167]$} & 0.079 & {$[-0.005-0.163]$} \\
\hline Rehabilitation, one more day & 0.075 & {$[-0.009-0.158]$} & 0.075 & {$[0.002-0.149]$} & 0.070 & {$[-0.003-0.143]$} \\
\hline Akaike information criteria & 67331 & & 67207.0 & & 67205.5 & \\
\hline
\end{tabular}

Cl: confidence interval; LOS: length of stay; LVH: low-volume hospital; IVH: intermediate-volume hospital; HVH: high-volume hospital; a: LOS*15 - 64 years; b: LOS*65 - 74 years; c: LOS*75 - 84 years; d: LOS*85 - years; e: LOS*TIA; f: LOS*lacunar; g: LOS*SAH; h: LOS*hemorrhage; I: LOS*subdural hematoma; j: LOS*infarction; k: LOS*RIND.

tries come to require longer LOS than expected.

Numerous studies have focused on the effectiveness and efficiency of healthcare, and healthcare outcomes such as LOS, mortality, and disability were the most important parameters when comparing quality of stroke care $[3,7,8]$. However, few studies have determined the essential impact 
Table 3. Factors Associated With Deterioration of Barthel Index

\begin{tabular}{|c|c|c|}
\hline Independent variables & Odd ratio & $95 \% \mathrm{CI}$ \\
\hline \multicolumn{3}{|l|}{ Age (for $15-64$ years) } \\
\hline $65-74$ years & 1.418 & {$[1.043-1.928]$} \\
\hline 75 - 84 years & 1.886 & {$[1.394-2.552]$} \\
\hline 85 years and over & 2.540 & {$[1.711-3.771]$} \\
\hline Male & 0.909 & {$[0.726-1.138]$} \\
\hline Transfer not at home & 6.402 & {$[4.993-8.207]$} \\
\hline Ambulance & 1.063 & {$[0.831-1.361]$} \\
\hline \multicolumn{3}{|l|}{ Principal Diagnosis (for TIA) } \\
\hline Lacunar & 1.576 & {$[0.552-4.497]$} \\
\hline $\mathrm{SAH}$ & 0.813 & {$[0.220-2.999]$} \\
\hline Hemorrhage & 0.981 & {$[0.325-2.963]$} \\
\hline Subdural hematoma & 1.860 & {$[0.542-6.380]$} \\
\hline Infarction & 1.627 & {$[0.578-4.581]$} \\
\hline RIND & 1.071 & {$[0.336-3.417]$} \\
\hline \multicolumn{3}{|l|}{ BI at admission (for dependent) } \\
\hline Assisted independence & 3.321 & {$[2.385-4.624]$} \\
\hline Independence with minimal assistance & 4.638 & {$[3.497-6.150]$} \\
\hline \multicolumn{3}{|l|}{ Charlson comorbidity index (for zero) } \\
\hline 1 & 1.003 & {$[0.759-1.325]$} \\
\hline 2 & 1.083 & {$[0.793-1.481]$} \\
\hline 3 & 1.228 & {$[0.782-1.930]$} \\
\hline 4 or more & 1.896 & {$[1.034-3.476]$} \\
\hline Cerebral vascular accident & 1.238 & {$[0.896-1.712]$} \\
\hline Dementia & 4.624 & {$[0.242-88.343]$} \\
\hline Atrial fibrillation or flutter & 1.047 & {$[0.711-1.544]$} \\
\hline Procedure-related complication & 2.308 & {$[0.881-6.047]$} \\
\hline Cognitive impairment after admission & 1.355 & {$[0.265-6.933]$} \\
\hline Deep vein thrombosis & 0.329 & {$[0.033-3.250]$} \\
\hline Procedure (reference; Medical) & 1.294 & {$[0.878-1.907]$} \\
\hline Ventilation & 1.146 & {$[0.502-2.619]$} \\
\hline \multicolumn{3}{|l|}{ Patient volume (reference; LVH) } \\
\hline IVH & 1.275 & {$[0.967-1.679]$} \\
\hline $\mathrm{HVH}$ & 1.357 & {$[1.036-1.777]$} \\
\hline \multicolumn{3}{|l|}{ Hospital (reference; Community) } \\
\hline Academic & 0.849 & {$[0.632-1.141]$} \\
\hline \multicolumn{3}{|l|}{ Fiscal year (for 2004) } \\
\hline 2005 & 1.396 & {$[0.838-2.325]$} \\
\hline 2006 & 1.101 & {$[0.660-1.838]$} \\
\hline 2007 & 1.289 & {$[0.765-2.172]$} \\
\hline 2008 & 1.168 & {$[0.690-1.979]$} \\
\hline LOS, one more day & 1.010 & {$[1.001-1.019]$} \\
\hline Initiation of rehabilitaion, one day delayed & 1.016 & {$[1.002-1.031]$} \\
\hline Rehabilitation, one more day & 1.004 & {$[0.991-1.016]$} \\
\hline Cox \& Snell $\mathrm{R}^{2}$, Nagelkerke $\mathrm{R}^{2}$ & $0.065,0.191$ & \\
\hline
\end{tabular}

$\mathrm{Cl}$ : confidence interval; RIND: reversible ischemic neurological deficit; SAH: subarachnoid hemorrhage; BI: Barthel index; LOS: length of stay; LVH: low-volume hospital; IVH: intermediate-volume hospital; HVH: high-volume hospital.

of LOS itself on functional status since Clarke's earlier critique of reducing LOS [5]. Longer LOS at acute care facilities, which relieve patient or family anxiety, might be due to disease severity, the use of many care processes includ- ing resource-intensive procedures, or individual practices $[5,18]$. Extended hospitalization undoubtedly separates patients from their usual daily life and may increase the risk of unwanted complications such as acquired infection [5]. 
Although our study was limited to patients with alert consciousness, we attempted to assess the reasons for variations in LOS for adequate adjustment of case mix as well as of all types of CVD. The results derived from this comprehensive study should provide valuable evidence to guide policy making to monitor unnecessary LOS, especially in advanced age groups because BI improvement was proven to be smaller with advanced age (Table 2).

Our study used BI as a continuous variable, though the difference from 55 to 65 was not equivalent to that from 25 to 35 because BI was originally an ordinal variable. Therefore, logistic regression was employed to measure the association of longer LOS with functional deterioration, which all models proved with the exception of insignificant results in intracranial hemorrhage and TIA. Delaying the initiation of rehabilitation, but not rehabilitation day, was likely to be associated with less BI improvement in Japanese acute care hospitals. The study hospitals might be more likely to focus their resources on critically ill patients in the earlier phases of hospitalization than on providing rehabilitation. Researchers should estimate the required LOS needed to recover from critical illness and consider the most appropriate time for initiating intensive rehabilitation in or out of acute care hospitals because hospital characteristics were not found to determine BI improvement in this study. It would be important to develop appropriate episode-based, cost-effective care strategies for initiating rehabilitation without any interruption of care. These community-based initiatives, when supported by electronic administrative databases of both acute and longterm care, would contribute to the enhancement of social welfare when available in other case mixes, such as degenerative musculoskeletal disorders or traumatic neurological damage. In addition, they would address any concerns of cost-shifting between hospitals and rehabilitation facilities or home and whether the incremental healthcare costs might balance the incremental gain in quality $[3,9,10]$.

We should mention several limitations of this study. First, data were collected from patients discharged from voluntarily participating hospitals over 4 consecutive months each year. This limits the generalizability of our results. However, the MHLW has gathered exhaustive data, and now plans to extend the data collection period to 12 months. Second, this study lacked information regarding the severity of cognitive impairment and time of onset of CVD, factors associated with functional status that are often utilized in health service research [1, 3, 6, 9]. The Japanese Stroke Databank (JSD) has collected such clinically informative data, and we may be able to obtain more meaningful findings using the administrative database in combination with the JSD [19]. Third, the causality between longer LOS and disease severity remains unresolved. We limited the study population to patients with no changes in consciousness to distill off the miasma regarding the association or causality between longer LOS and functional deterioration, and instead included variables proxy of disease severity such as ventilation use or complications. Other factors not included in this study might be related to disease severity and may explain some of the variation in LOS, which would be clarified in randomized, controlled studies or by community-based studies, if that factor is identified in an administrative database.

\section{Conclusions}

We have shown that LOS in acute care hospitals was independently associated with worsening functional status of CVD patients with alert consciousness at admission and at discharge. In addition, delaying rehabilitation and the day of rehabilitation was not conclusively associated with functional recovery, and the former determined the functional deterioration. Hospital patient volume and teaching status were not associated with improvements in BI.

To implement effective stroke care, stakeholders, including health service researchers and policy makers, should determine the appropriate LOS in acute CVD care and strengthen intensive and effective rehabilitation care either in or out of acute care settings.

\section{Competing Interests}

The authors declare that they have no competing interests.

\section{Acknowledgements}

This study was funded in part by Grants-in-Aid for Research on Policy Planning and Evaluation (Japanese Ministry of Health, Labor and Welfare, H19 seisaku-sitei 001).

\section{References}

1. Yoneda Y, Okuda S, Hamada R, Toyota A, Gotoh J, Watanabe M, Okada Y, et al. Hospital cost of ischemic stroke and intracerebral hemorrhage in Japanese stroke centers. Health Policy 2005;73(2):202-211.

2. Ministry of Health, Labour and Welfare Authority (2006). National Medical Care Expenditure (Estimates). [http://www.mhlw.go.jp/shingi/2006/09/s0906-6.html].

3. Kugler C, Altenhoner T, Lochner P, Ferbert A. Does age influence early recovery from ischemic stroke? A study from the Hessian Stroke Data Bank. J Neurol 2003;250(6):676-681.

4. Organisation for Economic Co-operation and Development (OECD). Health Data 2008 - Frequently Requested Data. [http://www.irdes.fr/EcoSante/DownLoad/ OECDHealthData_FrequentlyRequestedData.xls].

5. Clarke A. Why are we trying to reduce length of stay? 
Evaluation of the costs and benefits of reducing time in hospital must start from the objectives that govern change. Qual Health Care 1996;5(3):172-179.

6. Evans A, Harraf F, Donaldson N, Kalra L. Randomized controlled study of stroke unit care versus stroke team care in different stroke subtypes. Stroke 2002;33(2):449455.

7. Langhorne P, Taylor G, Murray G, Dennis M, Anderson C, Bautz-Holter E, Dey P, et al. Early supported discharge services for stroke patients: a meta-analysis of individual patients' data. Lancet 2005;365(9458):501-506.

8. Luk JK, Chiu PK, Chu LW. Rehabilitation of older Chinese patients with different cognitive functions: how do they differ in outcome? Arch Phys Med Rehabil 2008;89(9):1714-1719.

9. Rabadi MH, Rabadi FM, Edelstein L, Peterson M. Cognitively impaired stroke patients do benefit from admission to an acute rehabilitation unit. Arch Phys Med Rehabil 2008;89(3):441-448.

10. Saposnik G, Black SE, Hakim A, Fang J, Tu JV, Kapral MK. Age disparities in stroke quality of care and delivery of health services. Stroke 2009;40(10):3328-3335.

11. Andersson A, Levin LA, Oberg B, Mansson L. Health care and social welfare costs in home-based and hospital-based rehabilitation after stroke. Scand J Caring Sci 2002;16(4):386-392.
12. Appelros P. Prediction of length of stay for stroke patients. Acta Neurol Scand 2007;116(1):15-19.

13. Sundararajan V, Henderson T, Perry C, Muggivan A, Quan H, Ghali WA. New ICD-10 version of the Charlson comorbidity index predicted in-hospital mortality. J Clin Epidemiol 2004;57(12):1288-1294.

14. Zhan C, Miller MR. Administrative data based patient safety research: a critical review. Qual Saf Health Care 2003;12 Suppl 2:ii58-63.

15. Sulter G, Steen C, De Keyser J. Use of the Barthel index and modified Rankin scale in acute stroke trials. Stroke 1999;30(8):1538-1541.

16. World Health Organization. Health attainment, level and distribution in all Member States, estimates for 1997 and 1999. The World Health Report 2000. [http://www.who. int/whr/2000/en/annex05_en.pdf].

17. Organization for Economic Co-operation and Development (OECD). Health at a Glance - OECD indicators 2005. Paris: OECD Publishing, 2005:56.

18. Ishizaki T, Imanaka Y, Hirose M, Kuwabara K, Ogawa T, Harada Y. A first look at variations in use of breast conserving surgery at five teaching hospitals in Japan. Int J Qual Health Care 2002;14(5):411-418.

19. Kobayashi S. International experience in stroke registry: Japanese Stroke Databank. Am J Prev Med 2006;31(6 Suppl 2):S240-242. 